IdeAs

Idées d'Amériques

$17 \mid 2021$

Villes et culture dans les Amériques

\title{
The American Library in Paris : une institution hors du commun au service des lecteurs depuis cent ans
}

Audrey Chapuis

Traducteur : Anne Reynès-Delobel

(2) OpenEdition

Journals

Édition électronique

URL : https://journals.openedition.org/ideas/11114

DOI : $10.4000 /$ ideas. 11114

ISSN : 1950-5701

Éditeur

Institut des Amériques

Référence électronique

Audrey Chapuis, «The American Library in Paris : une institution hors du commun au service des

lecteurs depuis cent ans », IdeAs [En ligne], 17 | 2021, mis en ligne le 01 mars 2021, consulté le 03 juin 2021. URL : http://journals.openedition.org/ideas/11114; DOI : https://doi.org/10.4000/ideas.11114

Ce document a été généré automatiquement le 3 juin 2021

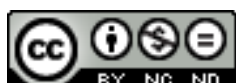

IdeAs - Idées d'Amériques est mis à disposition selon les termes de la licence Creative Commons Attribution - Pas d'Utilisation Commerciale - Pas de Modification 4.0 International. 


\title{
The American Library in Paris : une institution hors du commun au service des lecteurs depuis cent ans
}

\author{
Audrey Chapuis \\ Traduction : Anne Reynès-Delobel
}

\section{9 : Paris Library}

1 Un dimanche après-midi de l'automne 1919, près d'un an après la signature de l'armistice, une réunion est convoquée pour décider du sort de l'American Library ou de la « Paris Library », ainsi que la nomment parfois son personnel et ses habitués. Pour cette institution hybride, mi-entrepôt de transit, mi-bibliothèque de prêt, installée, de manière assez incongrue, dans un cadre fastueux, au 10, rue de l'Elysée, l'avenir est en effet des plus incertains.

2 Après de longs mois d'attente, on suppose que, comme les soldats pour lesquels elle a été conçue, la bibliothèque sera sous peu réacheminée vers les États-Unis. En effet, à bien des égards, même si elle dispose d'un catalogue exceptionnel, cette Paris Library n'est que l'une des 36 bibliothèques de camp érigées par le Library War Service (LWS), dans le cadre d'un projet d'envergure placé sous l'égide de l'American Library Association (ALA) qui a supervisé l'envoi de deux millions de livres et cinq millions de périodiques pendant le conflit, l'ensemble ayant transité par Paris avant d'être disséminé dans les bâtiments de fortune qui abritaient les quatre cent soixante-quatre bases militaires, camps d'entraînement et hôpitaux du corps expéditionnaire américain.

Depuis la fin du conflit, Paris s'est prise d'affection pour cette bibliothèque anglophone et les militaires américains ne sont plus les seuls à parcourir les rayonnages en libre-accès (ce système de consultation est alors très peu usité en Europe). On y croise aussi un grand nombre de Parisiens devenus des habitués des 
salles de lecture confortables où ils peuvent compulser les quotidiens de référence ainsi que des milliers d'ouvrages en anglais.

S'il n'existe jusqu'alors aucun plan préétabli pour donner à cette institution une forme pérenne, un projet a déjà été ébauché par ses administrateurs, notamment Burton Stevenson, bibliothécaire et romancier respecté, natif de l'Ohio, qui dirige le LWS en Europe. L'idée est de faire de l'American Library in Paris (ALP) non seulement une bibliothèque de prêt, mais aussi un mémorial vivant dédié aux soldats qui ont combattu pendant la guerre et un témoignage de l'action du LWS à laquelle la très grande majorité des bibliothèques publiques des États-Unis ont contribué. De l'avis de certains, le fonctionnement de l'ALP pourrait également servir d'exemple des méthodes de gestion scientifiques mises en place dans les bibliothèques américaines. On s'imagine ainsi que des bibliothécaires venus de l'Europe entière vont affluer vers l'ALP pour s'initier à la classification décimale de Dewey et découvrir les avantages de la consultation en libre-accès ainsi que d'autres fonctionnalités qui battent en brèche les méthodes établies.

5 La réunion prévue en ce dimanche va permettre d'évaluer l'importance que les Parisiens accordent réellement à la bibliothèque américaine. Les usagers vont-ils répondre présent? Et, le cas échéant, accepteront-ils de soutenir la campagne de financement? On estime en effet qu'une somme de cent cinquante mille francs est nécessaire pour garantir la solvabilité de l'ALP. L'ALA est-elle disposée à reconnaître le potentiel de cette dernière ? Comme le confie alors Stevenson dans son bref mais très beau rappel des origines de l'ALP : «l'avenir de la bibliothèque [est] plus qu'incertain ».

En ce jour d'automne, alors que le spectre de la guerre hante encore les esprits, une foule d'usagers ne tarde pas à se presser dans les locaux de la bibliothèque, impatiente de savoir quel sort on réserve à cette dernière. Après une série de discours par des partisans de son maintien, le premier à prendre la parole est un homme qui a joué un rôle de premier plan dans la création de l'ALP. Il se nomme Charles Seeger et il est le père du célèbre poète tombé au combat, Alan Seeger. Il s'engage à verser à la bibliothèque l'intégralité des droits d'auteur perçus sur la reproduction de l'œuvre de son fils. À sa suite, d'autres orateurs prennent la parole, notamment les représentants officiels de l'American Chamber of Commerce, l'American Woman's Club et l'American University Union, ainsi que divers défenseurs français de l'ALP, conservateurs de musées, professeurs de littérature et étudiants. Les promesses de dons d'argent affluent.

7 La somme collectée surprend tout le monde, car elle dépasse de loin l'objectif initial. De fait, elle incite l'ALA à poursuivre son engagement financier et à se porter acquéreur des locaux, et ouvre la voie à la constitution de la bibliothèque en société, en 1920. Une devise est alors choisie, Atrum Post Bellum, Ex Libris Lux (après les ténèbres de la guerre vient la lumière des livres).

\section{0 : une bibliothèque virtuelle}

Le 14 mars 2020, trois jours avant la fermeture par décret des bibliothèques et autres institutions culturelles en France, une réunion d'urgence réunissait le personnel de l'ALP pour discuter de la façon dont il convenait de réagir face au COVID-19. 
9 Un siècle après sa fondation, l'ALP compte près de cinq mille usagers représentant soixante nationalités, dont quarante pour cent d'Américains et vingt-deux pour cent de Français. Elle abrite le plus grand fonds de documents en langue anglaise du continent européen. Entièrement financée par la communauté, elle ne reçoit aucune dotation de l'État américain ou de l'État français (ceci étant, elle a pu bénéficier de subventions gouvernementales aux heures sombres de son histoire, notamment pendant la Grande Dépression, lorsqu'elle s'est vue octroyée cent mille francs par le gouvernement français). Aujourd'hui, l'ALP est autant un centre communautaire et culturel qu'une bibliothèque, et le terme "diversité » s'applique tout autant à ses usagers qu'à ses collections.

10 Chaque jour, on peut y voir des stylistes en train de feuilleter des revues de mode des années trente, des files de poussettes parquées dans les allées à l'heure où parents et enfants assistent au "Story Hour», et des grappes de lycéens qui bachotent dans la salle de lecture principale. Après s'être longtemps cherché une adresse permanente, la bibliothèque s'est installée en 1964 au 10, rue du Général Camou, une petite rue tranquille à deux pas de la Tour Eiffel. Son catalogue compte désormais près de cent mille volumes répartis sur trois étages et son système de prêt enregistre une moyenne de centquinze mille livres empruntés par an, soit le même total qu'à ses débuts. Les domaines les plus populaires sont la littérature pour jeunes adultes, la littérature adulte, les biographies, les guides de voyage, et les livres de cuisine et d'histoire. Les ouvrages imprimés sont complétés par des collections électroniques, mais le recours à l'imprimé est de loin supérieur à l'usage des ressources numériques.

$11 \mathrm{Au} \mathrm{xxI}$ siècle, face au déluge d'informations qui s'abat quotidiennement sur la population, la bibliothèque publique est souvent perçue comme un rempart. Ce n'est plus un lieu où l'on ne fait qu'emprunter des livres, mais un lieu de sociabilité et de débats citoyens. Désormais, l'agenda des animations que propose une bibliothèque est aussi important que ses collections. À titre d'exemple, aux États-Unis, le nombre de visites des bibliothèques publiques est en constante augmentation, même dans celles où le chiffre du prêt est en baisse. Si le COVID représente une menace tangible et imminente pour la santé publique, il met ainsi également en péril l'existence de nombreuses institutions culturelles dont la raison d'être se fonde dans les relations et les échanges interpersonnels.

12 Pendant la période du confinement, des bibliothécaires du monde entier se sont mis à réfléchir ensemble à la manière dont leurs institutions pouvaient poursuivre leur mission de service public alors même que leur accès était interdit aux usagers. En ce qui concerne l'ALP, au bout de quelques jours, les principaux services et programmes ont été mis en ligne. Pour lutter contre l'isolement, nous avons élaboré tout un programme d'activités et d'interactions en ligne. Avant la fin de la période de confinement, l'utilisation du numérique avait doublé.

13 Les fondateurs de l'ALP avaient été témoins des ravages causées par la guerre et l'épidémie de grippe de 1918. Ils savaient le réconfort que peut procurer un livre en période de crise. Dans le même esprit, nous avons lancé un projet intitulé At Home with the Library. Des comédiens et des écrivains connus se sont filmés en train de lire des extraits d'œuvres écrites par des écrivains de légende qui ont fréquenté la bibliothèque au fil de son histoire. En sus des lectures, d'autres vidéos faisaient revivre des bribes de la mémoire-palimpseste de la bibliothèque : dans l'une d'elles, Gertrude Stein et Alice B. Toklas se chamaillent entre deux rayonnages, dans une autre, Henry Miller écrit aux 
bibliothécaires pour obtenir des conseils de lecture sur le bouddhisme zen, dans une autre encore, on voit une Mary McCarthy très contrariée que l'ensemble de ses livres ne figure pas au catalogue de la bibliothèque. Ainsi, même si ses portes demeuraient closes au public, le lieu, tout bourdonnant de son riche passé, n'avait rien perdu de sa convivialité.

\section{0 : le lieu de tous les idéaux}

14 Charles Seeger, le mécène providentiel de l'ALP, devint le premier président de son conseil d'administration. Sa volonté était de faire de la bibliothèque un moyen d'améliorer l'entente entre les cultures et, plus particulièrement, de "lutter contre l'intolérance entre les races et les partis pris nationalistes [... en menant] la guerre contre l'ignorance et les préjugés ", ainsi que par la conviction qu'une population bien informée est davantage encline à « la compassion, la tolérance et la compréhension ».

15 Le conflit mondial avait donné à ces généreux idéaux un caractère d'urgence. Si la collecte de fonds pour la sauvegarde de la bibliothèque avait des visées pragmatiques (obtenir une dotation, trouver un bâtiment plus adapté, accroître les collections et conserver un personnel qualifié), elle poursuivait également un but qui pour n'être pas tangible n'en était pas moins pressant. Au XIX siècle, la fondation des bibliothèques publiques aux États-Unis s'était appuyée sur la conviction que l'éducation publique était au service du bien commun. Une génération plus tard, cette croyance, plus forte que jamais, était au cœur du projet de création de l'ALP. Ses fondateurs voyaient en cette institution indépendante un émissaire culturel et un lieu de mémoire supranational ou, selon les termes employés par un ambassadeur des États-Unis en France, « un sanctuaire à la fois invisible et impérissable ».

16 Ce zèle sincère n'était parfois pas dénué d'une certaine arrogance. En témoigne certaine description de la bibliothèque comme un messager d'une Nouveau Monde vers le Vieux Continent. Au fil des ans, certaines de ces nobles ambitions allaient se réaliser, d'autres ne seraient que de courte durée et d'autres encore ne verraient jamais le jour.

17 Dès le début, il avait été décidé d'une volonté commune que la bibliothèque serait administrée conjointement par des citoyens américains et français. De fait, si son conseil d'administration est resté majoritairement américain, son conseil consultatif est, quant à lui, majoritairement français et des personnalités telles que le Maréchal Joffre ou Raymond Poincaré y ont siégé. Ce dialogue interculturel est au fondement de la vision de la bibliothèque comme «messagère de paix » et creuset d'innovation pour la profession de bibliothécaire.

Burton Stevenson, autre figure fondatrice de l'ALP, a exercé les fonctions de directeur de 1925 à 1930 et on lui doit l'essor remarquable de la bibliothèque à cette période. La question du financement restait toutefois pressante et d'ingénieuses solutions ont été alors imaginées pour assurer l'approvisionnement en livres et le recrutement du personnel.

19 Dans le cadre d'un accord favorable à l'ensemble des parties, la bibliothèque met ainsi en place une collaboration avec soixante-cinq grandes maisons d'édition aux ÉtatsUnis, lesquelles lui font parvenir gratuitement des exemplaires de leurs nouvelles parutions qui sont exposées dans un espace dédié. Cette politique permet d'enrichir le catalogue d'environ mille titres par an, ce qui représente douze pour cent $(12 \%) \mathrm{du}$ 
total des acquisitions. Par ailleurs, ces acquisitions sont recensées et largement diffusées par le personnel de la bibliothèque qui montre un penchant certain pour la promotion publicitaire. En 1924, l'ALP se voit aussi dotée de sa propre revue littéraire, Ex Libris, qui comptera Ernest Hemingway et Gertrude Stein parmi ses contributeurs.

Une des grandes réussites de la bibliothèque est d'avoir noué des relations avec d'autres institutions. Grâce à son "Extension Service ", très moderne pour l'époque, elle devient ainsi le centre d'un service de prêt-entre-bibliothèques très actif qui dessert toute l'Europe. Elle entretient également des liens étroits avec le Comité américain pour les régions dévastées (CARD) qui administre conjointement avec l'ALA une formation en bibliothéconomie reconnue, dans les locaux de la bibliothèque. Par la suite, les étudiants formés à cette école contribueront à propager les méthodes que les fondateurs de l'ALP avaient souhaité partager avec le monde entier.

Afin d'enrichir ses collections, la bibliothèque noue des partenariats avec des organisations telles que la NAACP (National Association for the Advancement of Colored People) et l'American Association of University Women. Elle collabore également avec des institutions parisiennes, notamment la Bibliothèque nationale de France et la célèbre bibliothèque pour enfants L'Heure Joyeuse, avant de lancer son propre programme jeune public en 1928.

Quant au recrutement du personnel, il reflète la volonté de l'ALP de se doter de collaborateurs rompus aux méthodes américaines. C'est pourquoi la plupart d'entre eux est issue d'institutions américaines et détachée temporairement à Paris. La liste du personnel de l'époque ressemble à un annuaire de la profession. Dans celle de l'année 1930, par exemple, on peut relever les noms de Dorothy Reeder (Bibliothèque du Congrès), Helen A. Kirwin (New York Public Library), Doris Holt (Cleveland Public Library) et Mary Moser (Chicago Public Library). La bibliothèque a tiré avantage des compétences de ces collaborateurs hautement qualifiés.

Plus tard, Stevenson pourra déplorer que l'ALP n'ait pas atteint certains des objectifs qu'elle s'était fixés en matière de haute culture. En dépit de la fierté qu'inspiraient son service de référence et son "Extension Service ", l'établissement était en effet tiraillé entre sa volonté de servir un public d'érudits et l'attrait marqué des usagers pour les œuvres de fiction qu'on avait été jusqu'à remiser dans les étages du bâtiment de sorte à ne pas distraire l'attention des lecteurs des ouvrages plus érudits placés au rez-dechaussée. Stevenson lui-même professait un dédain non dissimulé pour les romans (ce qui peut paraître surprenant de la part d'un romancier) dont la présence était selon lui préjudiciable à la mission didactique et démocratique de l'ALP.

\section{0 : une bibliothèque au service de la communauté}

Organisation à but non lucratif indépendante et soutenue par la communauté, l'ALP a tout au long de son existence dû faire face à des défis institutionnels majeurs. À l'instar de la capitale qui l'abrite, elle a été le témoin d'un siècle de bouleversements politiques et sociétaux. Pour une bibliothèque, institution sociale par nature, le changement est une constante et la nécessité de s'adapter une obligation professionnelle. Patrimonialisation et innovation sont les deux facettes de sa mission ainsi que, comme l'actualité récente l'a montré, des enjeux cruciaux. 
Faire retour sur l'histoire de l'ALP, sur la ferveur humaniste de ses fondateurs et leur croyance dans l'entente entre les cultures et le bien-fondé d'une éducation populaire, est un réconfort dans ces temps d'immense incertitude. Les objectifs concrets poursuivis actuellement par la bibliothèque (notamment le prêt sécurisé et les programmes en ligne pour ceux qui doivent rester confinés) participent des mêmes visées. Dans ce contexte, les arguments enthousiastes avancés il y a cent ans en faveur de la création de l'ALP, tout comme la mobilisation de la communauté, ont une résonnance profonde.

\section{BIBLIOGRAPHIE}

American Library Association, « War Service Library Book ».

The American Library in Paris Yearbooks, 1922-1930.

Maack, Mary Niles, « American Bookwomen in Paris during the 1920s » Libraries \& Culture, vol. 40, $\mathrm{n}^{\circ}$ 3, Summer 2005, p. 399-415.

Maack, Mary Niles, « Americans in France: Cross-Cultural Exchange and the Diffusion of Innovations », The Journal of Library History (1974-1987), vol. 21, n² 2, Spring 1986, p.315-33.

Maack, Mary Niles, «"I Cannot Get Along without the Books I Find Here": The American Library in Paris during the War, Occupation, and Liberation, 1939-1945 ", Library Trends, vol. 53, n 3. December 2007, p. 490-512.

McCarthy, Justin, « In U.S., Library Visits Outpaced Trips to Movies in 2019 », GALLUP, January 24, 2020, In U.S., Library Visits Outpaced Trips to Movies in 2019 (gallup.com), consulté le 17 mars 2021.

Seeger, Charles, « The American Library in Paris: An Account of its Origin, Growth, and Purposes », New York, 1925.

Stevenson, Burton, « Some Reminiscences of the Paris Library," Library Journal, vol. 69, July 1944, p. 573-78.

Thomas, Susan Otis, « The American Library in Paris: An International Development in the American Library Movement », The Library Quarterly: Information, Community, Policy, vol. 34, $\mathrm{n}^{\circ} 2$, April 1964, p. 179-90.

Wright, Christopher, «April - And the American Library - In Paris », American Libraries 6, $\mathrm{n}^{\circ} 4$, April 1975, p. 226-27.

Young, Arthur P. Books for Sammies: The American Library Association and World War I, Beta Phi Mu chapbook, $\mathrm{n}^{\circ} 15,1981$. 


\section{AUTEURS}

\section{AUDREY CHAPUIS}

Audrey Chapuis est directrice de l'American Library in Paris depuis 2018. Elle a travaillé dans une vingtaine de bibliothèques, notamment la Widener Library de l'Université de Harvard et le Pritzker Legal Research Center de la Northwestern University School of Law, où elle était responsable du département des services d'accès. Elle est titulaire d'un master en bibliothéconomie et en sciences de l'information de l'Université de l'Illinois en 2006 et d'une licence de l'Université de Harvard. chapuis@americanlibraryinparis.org 